\title{
GASTOS SOCIAIS E O CRESCIMENTO ECONÔMICO DOS MUNICÍPIOS MINEIROS
}

SOCIAL EXPENDITURE AND THE ECONOMIC GROWTH

OF THE COUNTIES OF MINAS GERAIS

Priscila Ferreira de Paula

Marco Aurélio Marques Ferreira

Karla Maria Damiano Teixeira

RESUMO: Este artigo buscou identificar a relação existente entre os gastos sociais e o cresci mento econômico dos municípios de Minas Gerais. Especificamente, buscou-se analisar a influência dos gastos com assistência social, saúde, educação, cultura, urbanismo e saneamento sobre o crescimento do PIB em 2010. Além disso, verificou-se qual a melhor defasagem de tempo para avaliar a influência desses gastos, bem como o padrão de distribuição dos mesmos. Foi utilizado o modelo de regressão multinomial e o teste de média Kruskal-Wallis para amostras independentes, visto que os dados não atendem ao pressuposto da distribuição normal. Observou-se que os gastos com assistência social e cultura apresentaram uma relação negativa com o crescimento do produto, enquanto que os gastos com saúde, urbanismo e saneamento apresentaram uma relação positiva. Já os gastos relativos à educação não se mostraram estatisticamente significantes. Os resultados apurados podem orientar decisões políticas acerca da alocação de recursos públicos em áreas que apresentam relação com o crescimento ou decrescimento do PIB.

palavras-chave: Crescimento econômico, PIB, gastos sociais, gastos públicos, produtividade.

ABStRACT: This article aimed to identify the relationship between social spending and economic growth in the counties of Minas Gerais. Specifically, it sought to analyze the influence of spending on social assistance, health, education, culture, urbanism and sanitation on PIB growth in 2010. In addition, it was verified the best time lag to evaluate the influence of these expenditures, as well as the distribution pattern. The multinomial regression model and the Kruskal-Wallis mean test for independent samples were used, since the data do not meet the assumption of normal distribution. It was observed that spending on social assistance and culture had a negative relation with the growth of the product, while spending on health, urban planning and sanitation showed a positive relation. Education expenditures, however, were not statistically significant. The results found can guide policy decisions about the allocation of public resources in areas that are related to the growth or decrease of PIB.

KEYWORDS: Economic growth, PIB, social spending, public spending, productivity.

JEL Classification: H00, H50, I00. 


\section{INTRODUÇÃO}

A aplicação dos recursos públicos é um tema de grande relevância nos estudos de finanças públicas, sobretudo, pelo fato de se constituírem como principal meio para que o Estado exerça suas funções. A preocupação é recorrente na economia, principalmente em relação aos seus impactos sobre o crescimento econômico. Devido aos limites existentes para expansão da receita que financia o aumento dos gastos per capita, a sociedade exige, cada vez mais, uma melhor alocação dos recursos no atendimento das demandas sociais. Além disso, o aumento da produtividade é essencial para o crescimento econômico dos países, em especial, para aqueles em processo de estabilização (CÂNDIDo jr., 2001).

A grande ênfase dada ao ajuste fiscal e a busca por eficiência no setor público, pós década de 1990, são outros fatores que contribuíram para essa preocupação com a qualidade da alocação dos recursos públicos, uma vez que o Estado precisa lidar com as restrições financeiras e com as demandas sociais cada vez mais crescentes. Ribeiro (2008) explica que o uso eficiente dos recursos tem se tornado relevante, tanto em termos de boas práticas quanto de gestão fiscal. Segundo o autor, o próprio fenômeno da globalização gerou uma maior mobilidade de capital entre os países e, consequentemente, uma maior exigência quanto aos esforços tributários. Ademais, os próprios mecanismos legais de prestação de contas, como a Lei de Responsabilidade Fiscal promulgada nos anos 2000, são outros fatores que contribuem para a exigência de uma política fiscal de maior qualidade (RIBEIRO, 2008).

Como meio de mensurar a qualidade da gestão pública, torna-se necessário realizar estudos que permitam avaliar o impacto da aplicação dos recursos públicos no desenvolvimento social e econômico dos países. Nesse sentido, Cândido Jr. (2001) desenvolveu um trabalho com o objetivo de identificar a relação existente entre os gastos públicos e o crescimento econômico do Brasil no período entre 1947 e 1995. Suas análises apontaram que, no que se refere ao gasto total (incluindo os investimentos), os resultados indicaram uma externalidade positiva. No entanto, quanto ao diferencial de produtividade em relação ao setor privado, as externalidades foram negativas, indicando que o setor público apresentou $60 \%$ da produtividade do âmbito privado. Trabalho semelhante foi desenvolvimento por Ribeiro (2008), que buscou avaliar a eficiência do gasto público no Brasil comparada à um conjunto de países da América Latina. Seus achados, referentes ao período de 1998/2002, apontaram que os países que apresentaram melhor desempenho foram Costa Rica, Uruguai e Chile, sendo que o Brasil se apresentou na média quanto a avaliação dos serviços públicos, porém abaixo da média em relação à eficiência dos gastos.

Considerando a autonomia financeira dos municípios, desde o reconhecimento enquanto entes federados pela Constituição de 1988, é relevante analisar a alocação dos gastos públicos no âmbito local. Motta 
e Moreira (2007) explicam que os recursos públicos municipais servem de base para a melhoria da qualidade de vida de seus munícipes. Por meio da arrecadação de impostos e das transferências intergovernamentais, é possível financiar as responsabilidades dos municípios na prestação de serviços básicos como saúde, educação, entre outros (мотTA \& MOREIRA, 2007).

Dada a grande desigualdade social no Brasil e os problemas socioeconômicos resultantes, torna-se interessante estudar os gastos sociais, uma vez que impactam diretamente no Estado do bem-estar social. Além disso, considerando o fato de o país apresentar elevadas despesas governamentais e baixa eficiência na alocação dos recursos (CÂNDIDO JR., 2001), este trabalho teve como objetivo principal identificar a relação existente entre os gastos sociais e o crescimento econômico dos municípios de Minas Gerais. Além disso, o estudo também buscou identificar se altos dispêndios com serviços sociais têm relação direta com um alto nível de crescimento econômico, bem como verificar a melhor defasagem de tempo necessária para avaliar a influência desses gastos sobre a economia.

Para isso, a pesquisa se concentrou no estado de Minas Gerais, que se caracteriza como o estado brasileiro com maior número de municípios, totalizando 853 , além de corresponder a mais de $50 \%$ do total de municípios da região Sudeste. O estado ainda apresenta acentuado grau de desigualdade regional em termos de crescimento econômico. Dados da Fundação João Pinheiro (FJP), referentes ao ano de 2010, mostraram que enquanto regiões mais ricas como Central, Sul de Minas e Triângulo Mineiro apresentaram um percentual do Produto Interno Bruto (PIB) do estado correspondente a $47,5 \%, 12,4 \%$ e $11,1 \%$ respectivamente, regiões mais pobres como o Noroeste, Vale do Jequitinhonha e Mucuri e Norte apresentaram 1,7\%, 1,9\% e 3,9\% respectivamente (FUNDAÇÃO JOÃO PINHEIRO, 2012).

O trabalho se justifica pela necessidade crescente de eficiência na alocação dos recursos públicos, dadas as pressões orçamentárias enfrentadas pelos municípios no atendimento às demandas da população, além da margem reduzida para expansão das receitas. Motta e Moreira (2007) veem a administração municipal como uma unidade produtiva que transforma os gastos em uma infinidade de serviços públicos que vão impactar diretamente no nível de qualidade de vida. Trata-se de um processo lento e complexo que irá depender do estado inicial, do montante de gastos e da eficiência administrativa na gestão dos mesmos (MOTTA \& MOREIRA, 2007). No que tange aos gastos sociais, esses podem ser considerados o caminho para a erradicação da pobreza e da desigualdade social. Sendo assim, é importante analisar as áreas de investimentos que apresentam retornos positivos, tanto em termos econômicos quanto sociais, para que possam ser priorizadas pelos governos. 


\section{REVISÃO DE LITERATURA}

\section{GESTÃO E QUALIDADE DOS GASTOS PÚBLICOS}

Os gastos públicos realizados por uma determinada instância de governo para atender a um conjunto fixo de políticas públicas em um determinado ano fiscal, representam as preferências alocativas dessa própria administração (REZENDE, 1997). O autor classifica esses gastos em três categorias distintas, em função do fim último da ação dos governos, sendo elas: gasto mínimo, gasto social e gasto econômico. Os gastos mínimos representam a parcela dos gastos governamentais que são de uso exclusivo do governo, correspondentes às funções com o legislativo, judiciário, administração e planejamento, defesa e segurança. Estes gastos envolvem a provisão de bens e serviços considerados como bens públicos puros, uma vez que só podem ser provisionados exclusivamente pelo governo (REZENDE, 1997).

Já os gastos sociais são aqueles destinados à provisão de bens e serviços considerados semipúblicos, pois, nesse caso, o governo não assume uma posição de monopólio, abrindo espaço para a atuação dos mecanismos de mercado. Esses gastos são dados na função educação, cultura, saúde, saneamento, assistência, previdência, habitação e urbanismo. Os gastos econômicos são destinados às atividades econômicas, nas quais não há a necessidade de envolvimento do governo. No entanto, na prática, o governo atua nessas atividades como forma de regular o mercado ou intervir no desempenho empresarial. Esses gastos são dados pelas funções agricultura, comunicações, desenvolvimento regional, energia e recursos minerais, indústrias, comércio e serviços, relações exteriores, trabalho e transporte (REZENDE, 1997).

Esses gastos podem ser considerados produtivos quando conseguem atingir os objetivos propostos com um menor nível de custo possível. Já os gastos improdutivos são dados pela diferença entre o gasto efetivo e aquele que apresentar o menor custo para um mesmo objetivo. Dentre os diversos fatores que afetam negativamente a qualidade dos gastos públicos, é possível citar a falta de qualificação técnica do pessoal, as deficiências do processo orçamentário, problemas com corrupção, incertezas, paralisia de obras, entre outros. Soma-se a isso, a própria tendência natural que induz a um crescimento mais rápido dos gastos públicos se comparado à arrecadação tributária (CÂNDIDO JR., 2001).

Segundo Alonso (1999), dada a dificuldade existente em se aumentar a carga tributária, a tendência pós década de 1980 foi de se concentrar na redução dos gastos. No entanto, organismos internacionais como o Banco Mundial e o Fundo Monetário Internacional (FMI) vêm incentivando, para além do corte de gastos, a preocupação com a qualidade dessas alocações, pois a redução das despesas, além de comprometer a qualidade dos serviços prestados, também impacta na capacidade competitiva dos países (ALONSO, 1999). 
Para o autor, a preocupação com esse controle dos gastos públicos no Brasil se intensificou a partir de 1986, quando foi criada a Secretaria do Tesouro Nacional, do Ministério da Fazenda, juntamente com o Sistema Integrado de Administração Financeira (SIAFI). Isso representou um avanço significativo para a gestão das finanças públicas brasileiras, fazendo com que o país se tornasse referência internacional no controle informatizado de gastos ainda no início dos anos de 1990. No entanto, sistemas desenvolvidos no país, como o SIAFI, têm por finalidade controlar a execução das despesas e não apurar os custos dos serviços públicos. Com isso, o governo não tem conhecimento claro de quais atividades agregam maior valor ao resultado final das ações, nem em que medida cada uma das despesas irá contribuir para os resultados obtidos. Isso prejudica a identificação de onde há desperdício e onde há eficiência na alocação, sendo que o desconhecimento desses custos pode ser considerado o maior indicativo de ineficiência no provisionamento dos serviços públicos (ALONSO, 1999).

Cândido Jr. (2001) explica que existem diversas dificuldades que prejudicam a mensuração correta da produtividade desses gastos, uma vez que, para isso, é necessário avaliar todos os benefícios dos programas, bem como os custos de oportunidade do processo. Isso implica identificar todos os objetivos primários de cada programa de gasto, eliminando da análise todos os esforços referentes a objetivos secundários. Evita-se, assim, dispersões e desperdícios, concentrando-se apenas na finalidade principal de cada gasto público. Dentre as medidas que impactam diretamente na produtividade dos gastos, podem ser citadas: a redução de gastos com pessoal por meio de cortes na folha de pagamento pode gerar uma deterioração da qualidade dos serviços prestados; a má alocação de subsídios e transferências governamentais pode impactar na eficiência dos programas; os investimentos devem ser alocados em setores que geram maiores externalidades positivas e devem ser complementados por investimentos privados (CÂNDIDO JR., 2001).

\section{GASTOS PÚBLICOS E CRESCIMENTO ECONÔMICO}

A reforma tributária resultante da Constituição de 1988 provocou um processo de municipalização das finanças públicas, sobretudo da receita e do gasto, graças à forte onda de descentralização fiscal que culminou em uma maior participação dos estados e municípios na administração pública nacional. A consolidação e aprofundamento do processo de descentralização, resultante das mudanças trazidas pela Constituinte, permitem considerar o Brasil como uma Federação acentuadamente descentralizada, inclusive com seu grau de autonomia fiscal se sobressaindo se comparado a outros países (AFONSO \& ARAÚJO, 2001).

Segundo Rocha e Giubert (2007), o efeito da política fiscal sobre o crescimento econômico é objeto de constante debate, tanto na literatura internacional quanto nacional. Modelos como os de demanda agrega- 
da keynesiano indicam relações simples entre o orçamento público e a atividade econômica, em que um corte no déficit do governo reduziria o consumo e o produto. Esse corte poderia ser por meio da redução de gastos ou aumento dos tributos. No entanto, eles nem sempre foram condizentes com a realidade, uma vez que, em muitos países, os cortes advindos dos programas de estabilização da economia resultaram em expansões e não contrações fiscais (ROCHA \& GIUBERT, 2007).

Essa constatação deu início ao período conhecido como "visão expectacional da política fiscal", pois partia da percepção de que os modelos tradicionais keynesianos não consideravam o aspecto da sinalização do aumento dos gastos ou cortes nos impostos. Essa visão compartilhava da ideia de que o efeito de um aumento no gasto público dependeria de um consequente aumento das obrigações futuras de impostos. Isso significa que, os indivíduos reagem a essas mudanças alterando suas distribuições de probabilidade para gastos e impostos futuros. Um exemplo claro desse efeito pode ser observado em uma situação de grandes aumentos no gasto público e, sendo assim, impostos consequentemente mais altos. Nesse caso, isso reduziria o consumo privado e o resultado seria um efeito contracionista da política fiscal. Em contrapartida, se pequenos aumentos temporários são revertidos no futuro, isso não afetaria esse consumo privado e, portanto, o efeito não seria o mesmo (FELDSTEIN, 1982 apud ROCHA \& GIUBERT, 2007).

Cândido Jr. (2001) aponta que Wagner (1980) foi um dos pioneiros economistas a estudar a relação existente entre gastos públicos e crescimento econômico. A hipótese de Wagner, conhecida como "Lei dos Dispêndios Públicos Crescentes", defende a ideia de que o crescimento da renda per capita estaria ligado à uma participação cada vez maior do governo na promoção de bens públicos. Essa proposição estaria baseada em aspectos como: 1) $\mathrm{O}$ aumento da renda implicaria em um aumento das demandas por bens públicos; 2) Mudanças demográficas como, por exemplo, redução da mortalidade, demandaria maiores gastos com a população idosa; 3) Altas taxas de natalidade e de população jovem demanda maiores dispêndios com educação; e 4) Programas sociais como os de redistribuição de renda, seguridade, entre outros, aumenta a importância das transferências para o orçamento público (cândido jr., 2001).

Ainda segundo o autor, a discussão mais recente sobre a influência dos gastos públicos no crescimento econômico é resultante das teorias de crescimento endógeno. Ao contrário dos modelos de crescimento neoclássicos tradicionais, como o de Solow, que tratavam de maneira exógena a política fiscal e as mudanças tecnológicas e demográficas, as teorias recentes incluem essas variáveis nos modelos, uma vez que elas podem implicar em um diferencial de crescimento, expandindo o período de convergência entre as rendas dos países (A, 2001).

É possível observar uma série de estudos empíricos que encontraram relações positivas e negativas entre os gastos públicos e o crescimento econômico. Na literatura internacional, Devarajan et al. (1996) 
tiveram por objetivo identificar quais componentes dos gastos públicos apresentam resultados produtivos em um conjunto de 43 países em condições de desenvolvimento. Os resultados apontaram que mesmo os gastos considerados produtivos podem se tornar improdutivos quando são realizados em excesso. Os gastos com saúde, educação, transporte, comunicação e capital apresentaram esse efeito e somente os gastos correntes impactaram positivamente no crescimento econômico. Segundo os autores, esse resultado está relacionado ao fato de os países em desenvolvimento concentrarem seus dispêndios em capital e, não, em custeio.

Herrera \& Blanco (2004) buscaram identificar as principais tendências da política fiscal no Brasil, no período entre 1999 e 2005. O trabalho utilizou duas abordagens complementares para analisar a interação entre as variáveis: um único método da equação (a ARDL) e um múltiplo método da equação (VAR cointegratng). Os resultados mostram que a política fiscal no Brasil pode ser considerada policíclica no curto prazo e anticíclica no longo prazo, apresentando efeitos negativos dos subsídios sobre o crescimento econômico.

Já Pang \& Herrera (2005) procuraram avaliar a eficiência das despesas públicas para 140 países entre o período de 1996 e 2002. Foi realizada a estimativa da eficiência dos gastos públicos por meio das técnicas de Análise Envoltória de Dados (AED) e da Free Disposable Hull (FDH). Os resultados apontaram uma associação negativa entre o tamanho das despesas públicas e os indicadores de eficiência, entre a porcentagem da massa salarial e o orçamento e eficiência total, entre a proporção de serviços que é financiado publicamente e os indicadores de eficiência, e, entre o financiamento externo e a eficiência. Já a urbanização apresentou uma associação positiva com a eficiência. Esses resultados corroboram com os achados de Devarajan et al. (1996) que indicaram que aumentos excessivos nos gastos públicos podem contribuir para torná-los improdutivos.

No Brasil, Cruz et al. (2010) realizaram um trabalho com o intuito de avaliar a eficiência dos gastos públicos em infraestrutura e capital humano sobre o crescimento econômico e a redução da pobreza. Os dados foram analisados para o período entre 1980 e 2007, utilizando o método de equações simultâneas, buscando-se identificar as interações diretas e indiretas entre as variáveis endógenas e exógenas. Os resultados apontaram que os gastos em infraestrutura e capital humano contribuíram para o crescimento econômico com redução de pobreza. Além disso, as análises apontaram que despesas com educação, saúde, estrada e energia afetam positivamente o rendimento per capita e a produtividade da economia.

Na mesma linhagem, Morais et al. (2012) buscaram identificar a influência dos gastos públicos no crescimento econômico de 73 municípios do Ceará, entre 2002 e 2005. Como variável dependente foi utilizado o PIB per capita e, como variáveis independentes, as despesas por função (administração e planejamento, legislativo, custeio com 
pessoal, assistência e previdência social, saúde e saneamento), o consumo de energia elétrica industrial e o número de pessoas com ensino fundamental completo. As análises apontaram que investimentos em capital físico contribuem para o aumento do PIB, enquanto que despesas administrativas e de planejamento acarretariam em decréscimo. Além disso, as despesas com saúde e saneamento apresentaram coeficiente significativo e negativo. Já as despesas com legislativo e custo de pessoal foram significativas apenas em nível de $10 \%$.

\section{METODOLOGIA}

\section{BASE DE DADOS}

Os dados foram escolhidos com base no objetivo de se analisar a relação entre os gastos sociais e o crescimento econômico dos municípios. Por gastos sociais, foi considerada a classificação de Rezende (1997) que considera esse grupo formado pelas despesas na função educação, cultura, saúde, saneamento, assistência, previdência, habitação e urbanismo. Essas despesas foram coletadas através do banco de dados Finanças do Brasil (FINBRA) para os anos de 2009 e 2008, permitindo assim, verificar a melhor defasagem de tempo. Para o crescimento econômico foi utilizada a proxy 'Produto Interno Bruto', disponibilizada pelo Instituto Brasileiro de Geografia e Estatística (IBGE) para o ano de 2010. Todas as despesas foram transformadas em valores per capita e representam variáveis métricas.

Inicialmente, a amostra foi composta pelos 853 municípios mineiros, mas dada a alta discrepância da variável dependente 'PIB', principalmente em função de regiões que atuam na atividade de mineração, foram excluídos 21 municípios que se caracterizaram como outliers, o que resultou em uma amostra de 832 observações. Além disso, a despesa na função habitação não foi considerada na análise, pois mais de $50 \%$ dos municípios estavam com esses valores ausentes no banco de dados do FINBRA. Outra despesa excluída foi a previdência social, que como será abordada adiante, apresentou problemas de multicolineariedade no modelo de regressão. Sendo assim, as variáveis utilizadas neste estudo foram:

$$
\begin{aligned}
& X_{1}=\text { Assistência Social per capita } \\
& X_{2}=\text { Saúde per capita } \\
& X_{3}=\text { Cultura per capita } \\
& X_{4}=\text { Educação per capita } \\
& X_{5}=\text { Urbanismo per capita } \\
& X_{6}=\text { Saneamento per capita } \\
& X_{7}=\text { PIB per capita }
\end{aligned}
$$

\section{REGRESSÃO MULTINOMIAL}

A técnica utilizada para identificar os gastos sociais que possuem relação com o crescimento econômico consistiu na regressão logit mul- 
tinomial. Segundo Maroco (2011), esse modelo é utilizado quando a variável dependente é nominal e politômica, ou seja, além da variável dependente ser de natureza categórica, apresenta mais de duas classes mutualmente exclusivas. Caso a variável dependente apresente apenas duas classes, emprega-se o uso da regressão binomial. Neste estudo, a variável dependente será o PIB per capita classificado em três categorias ou classes: baixo, médio e alto. Essas categorias foram definidas conforme um corte realizado em função em função da média $\pm 1 / 2$ desvio padrão, conforme será mostrado adiante.

Basicamente, o modelo de regressão multinomial consiste em um conjunto de $\kappa$ modelos logísticos corrigidos, sendo um para cada k classe da variável dependente, aqui definidas como faixas de crescimento econômico. A probabilidade da variável depende $\mathrm{Y}$ em relação a cada uma das classes, pode ser descrita da seguinte forma matricial (MAROCO, 2011):

$$
\begin{array}{r}
P(Y=0 \mid X)=\frac{e^{X \beta 0}}{e^{X \beta 0}+e^{X \beta 1}+e^{X \beta 2}} \\
P(Y=1 \mid X)=\frac{e^{X \beta 1}}{e^{X \beta 0}+e^{X \beta 1}+e^{X \beta 2}} \\
P(Y=2 \mid X)=\frac{e^{X \beta 2}}{e^{X \beta 0}+e^{X \beta 1}+e^{X \beta 2}}
\end{array}
$$

A generalização do modelo logístico para variáveis com número de níveis acima de $2(k>2)$ é direta e pode ser empregada para $\kappa$ classes. A probabilidade de uma observação $X$ pertencer a uma das classes de $\mathrm{y}_{1}$ dada pela seguinte equação (BITTENCOURT, 2003):

$$
\left.P\left(\mathrm{Y}=\mathrm{y}_{1}\right) \mid \mathrm{X}\right)=\frac{\exp \backslash\left\{\mathrm{g}_{\mathrm{i}}(\mathrm{x}) \backslash\right\}}{1+\sum_{\mathrm{j}=1}^{\mathrm{k}-1} \exp \backslash\left\{\mathrm{g}_{\mathrm{j}}(\mathrm{x}) \backslash\right\}} \quad \begin{aligned}
& \mathrm{i}=1,2, \ldots, k-1
\end{aligned}
$$

A regressão logística apresenta dois testes importantes para avaliar a significância do modelo. O primeiro é o teste da razão de verossimilhança, no qual se testa a hipótese de que pelo menos um dos parâmetros é diferente de zero $\beta_{\mathrm{ij}} \neq 0{ }_{i}$. Esse valor é resultado da comparação entre o valor da função de verossimilhança para o modelo apenas com o intercepto e para o modelo final com todos os parâmetros estimados. O segundo teste é o de Wald, definido como o quadrado da razão entre o máximo estimado de verossimilhança para o coeficiente e o seu erro padrão, em que a hipótese nula a ser testada é que o particular coeficiente $\beta_{\mathrm{ij}}$ é igual a zero (BITTERNCOURT, 2003). Os testes foram empregados para verificar o ajustamento do modelo e identificar quais despesas se apresentaram estatisticamente significativas para a logit do crescimento econômico. 
De acordo com alguns autores, é difícil se determinar ao certo, a época em que o modelo logístico foi utilizado pela primeira vez. Alguns deles concordam que o emprego do método ganhou reconhecimento após o trabalho de Truett, Cornfield e Kannel (1967) que buscaram analisar o risco de doença coronária. Nesse sentido, esse estudo é considerado como marco inicial dos trabalhos com a regressão logística nas áreas de saúde (COX \& SNELL, 1989; HOSMER \& LEMESHOW, 1989; apud BITTENCOURT, 2003).

A técnica, inicialmente, foi desenvolvida para variáveis de respostas binárias e, posteriormente, foi estendida para variáveis de respostas politômicas. O uso da técnica se expandiu rapidamente, graças a amplitude de áreas na qual pode ser empregada, sendo a saúde, uma das pioneiras. Além da saúde, também é possível encontrar seu uso no campo da econometria, administração, educação, entre outros, o que contribui para a importância do método como ferramenta estatística (BITTENCOURT, 2003).

\section{TESTE DE KRUSKALL-WALLIS}

Além do modelo de regressão multinomial, o trabalho também empregou o teste de Kruskal-Wallis para identificar os parâmetros de distribuição das três faixas de crescimento econômico. Trata-se de uma teste estatístico não-paramétrico que deve ser empregado em variáveis de escala pelo menos ordinal e se constitui como uma alternativa ao teste One-Way Anova, quando os dados não atendem aos pressupostos deste método, que são a normalidade e a igualdade das variâncias. Além disso, a ferramenta busca testar a igualdade das variáveis em relação a um mesmo parâmetro de localização (PESTANA \& GAGEIRO, 2008).

Pestana e Gageiro (2008) apontam que o objetivo do teste é verificar a hipótese de existência de um parâmetro comum para as k populações e a hipótese alternativa de que pelo menos uma das observações apresenta valores diferentes do parâmetro apresentado. Portanto, o teste foi empregado para verificar se as diferentes faixas de crescimento econômico apresentam a mesma tendência de distribuição para os gastos sociais. As hipóteses a serem testadas pelo método são dadas por (PESTANA \& GAGEIRO, 2008, p. 458):

$H_{0}$ As k distribuições têm o mesmo parâmetro de localização.

$H_{1}$ Pelo menos uma das k populações têm um parâmetro de localização superior ou inferior ao das outras.

Além disso, o teste de Kruskal-Wallis é definido pela seguinte equação (PESTANA \& GAGEIRO, 2008, p.458):

$$
\begin{aligned}
& H=\frac{12}{n(n-1)} \times \sum_{j}^{k} \frac{R_{j^{2}}}{n_{j}}-3 \times(n+1) \text {, caso não haja empates } \\
& H_{E}=\frac{H}{\sum_{j}^{k}\left(t_{i^{3}}-t_{i}\right)} \\
& n^{3}-n
\end{aligned}
$$


Em que $n$ é igual a dimensão da amostra, $k$ é igual ao número de grupos, $R j$ corresponde as ordenações da categoria $j$ e tj é igual ao número de observações empatadas na categoria i. O teste se baseia nas soma das ordens dos grupos de maneira crescente, sendo a menor observação atribuída o valor 1 , a seguinte 2 e assim, sucessivamente. Caso haja empate (mesmo valor), é atribuído o valor médio pela posição que lhe corresponderia se não houvesse empate (PESTANA \& GAGEIRO, 2008).

\section{RESULTADOS}

A tabela 1 fornece as estatísticas descritivas das variáveis utilizadas no modelo de regressão. Pode ser observado que as variáveis 'Assistência Social', 'Cultura', 'Urbanismo' e 'Saneamento' apresentaram uma média de $\mathrm{R} \$ 47,09, \mathrm{R} \$ 27,78, \mathrm{R} \$ 118,11, \mathrm{R} \$ 33,89$, respectivamente, com desvio padrão indicando alta dispersão dos dados. Já as variáveis 'Saúde' e 'Educação', apresentaram média de $\mathrm{R} \$ 350,20$ e $\mathrm{R} \$ 367,26$, respectivamente, com dispersão moderada dos dados em torno da média. E, a variável dependente 'PIB' apresentou uma média de $\mathrm{R} \$ 9.677,26$ com desvio padrão indicando alta discrepância. Considerou-se alta discrepância dos valores para um desvio padrão superior à metade da média. Já as medidas de assimetria e curtose indicaram, para todas as variáveis, que os dados se distribuem de maneira assimétrica à direita e leptocúrtica (em cume).

TABELA 01 - Estatística Descritiva

\begin{tabular}{c|c|c|c|c|c|c|c} 
& $\mathrm{N}$ & MíNIMO & MÁxIMO & MÉDIA & $\begin{array}{c}\text { DESVIO } \\
\text { PADRÃO }\end{array}$ & ASSIMETRIA & CURTOSE \\
\hline $\begin{array}{c}\text { ASSISTÊNCIA } \\
\text { SOCIAL }\end{array}$ & 826 & 3,57 & 371,16 & 47,09 & 34,94 & 3,38 & 20,70 \\
\hline SAÚDE & 826 & 147,93 & 1509,34 & 350,21 & 138,36 & 2,59 & 13,04 \\
\hline EDUCAÇÃO & 826 & 82,21 & 1464,89 & 367,27 & 146,00 & 1,84 & 6,89 \\
\hline CULTURA & 816 & 0,06 & 291,97 & 27,78 & 28,33 & 2,60 & 12,20 \\
\hline URBANISMO & 820 & 0,65 & 648,71 & 118,12 & 80,12 & 1,93 & 6,14 \\
\hline SANEAMENTO & 729 & 0,01 & 309,82 & 33,90 & 44,63 & 2,31 & 6,44 \\
\hline PIB & 832 & 3253,54 & 41378,26 & 9677,08 & 6281,82 & 2,23 & 5,91
\end{tabular}

FONTE: Resultados da pesquisa.

Como o modelo de regressão logística multinomial é aplicado para uma variável dependente nominal e acima de duas classes (maroco, 2011), foi criada a variável qualitativa 'faixa PIB' classificando os municípios em três grupos de crescimento econômico: 'baixo', 'médio' e 'alto'. Para isso, foi considerado o critério da média $\pm 1 / 2$ desvio padrão, para deixar a distribuição entre as faixas um pouco mais uniforme do que se fosse considerado 1 desvio. A tabela a seguir indica os intervalos de crescimento econômico, em função do PIB per capita: 
TABELA 02 - Faixas de crescimento econômico

\begin{tabular}{c|c|c} 
CRITÉRIO & ESCORES & CLASSIFICAÇÃO \\
\hline $\begin{array}{c}\text { Inferior à média }(1 / 2 \\
\text { desvio-padrão abaixo })\end{array}$ & $\mathrm{E}<6536,17$ & Baixo \\
\hline Entre $+/-1 / 2$ desvio-padrão & $6536,17<\mathrm{E}<12817,99$ & Médio \\
\hline $\begin{array}{c}\text { Superior à média }(1 / 2 \\
\text { desvio-padrão acima })\end{array}$ & $\mathrm{E}>12817,99$ & Alto
\end{tabular}

FONTE: Resultados da pesquisa.

Considerando que um dos pressupostos da regressão logística é que a distribuição dos dados não é normal (não-paramétrica), foram realizados os testes de Kolmogorov-Smirnov e Shapiro-Wilk para todas as variáveis selecionadas. O resultado do sig $<0,05$ para todas as variáveis permite rejeitar a hipótese nula de que a distribuição é normal, o que justifica empregar métodos para dados não-paramétricos neste estudo.

Foram realizadas regressões para duas defasagens de tempo $(t$ - 1) e ( $t$ - 2), buscando identificar qual seria o melhor modelo de análise. A análises para as duas defasagens de tempo não apresentaram diferenças significativas, sendo que, os resultados encontrados semelhantes para os dois modelos. No entanto, optou-se pela defasagem de tempo $(t-1)$ que apresentou, mesmo de maneira marginal, uma melhor qualidade de ajuste do modelo se comparada ao primeiro, observada por meio do pseudo $\mathrm{R}^{2}$. Dessa forma, optou-se por seguir as análises com o modelo de regressão em que as despesas por função social estão defasadas em 1 ano (2009) em relação ao PIB per capita (2010).

Considerar a defasagem entre os gastos públicos e o grau de crescimento econômico é importante, uma vez que os dispêndios realizados no decorrer de um exercício só apresentarão resultados nos períodos posteriores. Além disso, como explica Cândido Jr. (2001), uma mensuração ideal da produtividade desses investimentos deve levar em contas todos os benefícios gerados pelos programas, sendo assim, respeitar esse prazo é interessante pois a maioria dos programas resultantes de uma política pública são desenvolvidos para gerarem resultados em um período de médio à longo prazo.

Conforme mencionado anteriormente, a despesa na função habitação foi excluída, uma vez que este valor estava indisponível no banco de dados do FINBRA para a maior parte dos municípios. Além disso, numa análise inicial, foi observado que a despesa com previdência social apresentava problemas de multicolineariedade com a assistência social, visto que a correlação entre as duas é forte, o que interferiu na qualidade de ajuste do modelo. Dessa forma, optou-se por retirar a variável 'previdência social' pois esta apresentava uma menor significação. Bittencourt (2003) explica que esses é um dos problemas enfrentados pela regressão logística quando há a presença de forte correlação entre as variáveis explicativas. 
Das 832 observações, foi identificado que 38,4\% dos municípios de Minas Gerais se enquadram na faixa de crescimento econômico 'baixo', 42,2\% na faixa 'médio' e 19,4\% na faixa 'alto'. Esse cenário pode estar associado ao fato do Brasil apresentar uma tendência de elevados gastos governamentais associada a baixa eficiência na alocação dos mesmos, o que acaba contribuindo paras torná-los improdutivos, como foi explicado por Cândido Jr. (2001). Além disso, a análise do crescimento econômico também deve incorporar outras variaríeis que antes eram tratadas de maneira exógena, como mudanças tecnológicas e demográficas, que podem explicam essas diferenças (CÂNDIDO JR., 2001). No caso de Minas Gerais, é importante considerar que regiões mineradoras, como o centro-sul, acabam agregando um diferencial no PIB se comparadas às demais, além do fato do estado ser composto, em sua maioria, por cidades de pequeno porte, o que explica o crescimento econômico estar concentrado na faixa de baixo e médio.

Na regressão logística, a qualidade de ajuste do modelo é dada pelo teste da rácio da verossimilhança entre o modelo nulo e o final (MAROCO, 2011). Sendo assim, o teste do Qui-quadrado $=102,405$ para um sig $=0,00$, possibilitou rejeitar a hipótese nula de que o modelo não é significativo, indicando que pelo menos uma das variáveis independentes influencia o crescimento econômico dos municípios. Os valores do AIC e do BIC também apresentaram um melhor ajustamento do modelo final em relação ao intercepto, reforçando assim, o ajuste do modelo, como mostra a tabela 3:

TABELA 3 - Informações de ajuste do modelo

\begin{tabular}{c|c|c|c|c|c|c}
\multirow{2}{*}{ MODELO } & \multicolumn{3}{|c|}{$\begin{array}{c}\text { CRITÉRIOS DE AJUSTE } \\
\text { DE MODELO }\end{array}$} & \multicolumn{3}{|c}{$\begin{array}{c}\text { TESTES DE RAZÃO DE } \\
\text { PROBABILIDADE }\end{array}$} \\
\cline { 2 - 7 } & AIC & BIC & $\begin{array}{c}\text { Probabilidade } \\
\text { de log }-2\end{array}$ & $\begin{array}{c}\text { Chi- } \\
\text { quadrado }\end{array}$ & df & Sig. \\
\hline $\begin{array}{c}\text { APENAS } \\
\text { INTERCEPTAÇÃO }\end{array}$ & 1507,404 & 1516,552 & 1503,404 & & & \\
\hline FINAL & 1428,999 & 1493,031 & 1400,999 & 102,405 & 12 &, 000
\end{tabular}

FONTE: Resultados da pesquisa.

Além disso, as medidas do Qui-quadrado e da deviance também permitem analisar esse ajustamento (MAROCO, 2011). Para um Quiquadrado $=1482,01$ e sig $=0,116$ e deviance $=1400,99$ e sig $=0,621$, não se rejeita a hipótese nula de que o modelo se ajusta propriamente aos dados. Portanto, é possível concluir que o modelo apresenta um bom ajustamento.

Já o pseudo $\mathrm{R}^{2}$ apresenta a estimativa dos pseudo- $\mathrm{R}^{2}$ de Cox e Snell, Nagelkerke e McFadden de 0,133, 0,152 e o,68 respectivamente. Como aponta Maroco (2011), o McFadden é o que melhor indica o ajustamento do modelo, sendo assim, é possível concluir que o modelo apresenta um ajustamento baixo, indicando que 6,8\% do PIB (crescimento econômico) dos municípios é explicado pela relação entre os gastos com 
assistência social, saúde, educação, cultura, urbanismo e saneamento. Já Bittencourt (2003) dá preferência ao Nagelkerke, pois segundo o autor esse teste apresenta uma medida no intervalo [0,1], sendo assim, o poder de explicação do modelo seria de $15,2 \%$. Cabe destacar que, o trabalho tem por finalidade verificar a existência de relação entre o crescimento econômico e os gastos sociais, e não explicar o crescimento econômico em si, pois, para isso, seria necessário a inclusão de outras variáveis que sejam determinantes para o PIB, o que não é o foco do artigo.

O teste de rácio de verossimilhança para cada uma das variáveis do modelo indicou que a variável 'assistência social' apresentou um efeito estatisticamente significativo $\left(\mathrm{G}^{2}=28,43\right.$ e sig $\left.=0,00\right)$ sobre a logit do crescimento econômico, considerando um intervalo de confiança de $5 \%$. Na mesma linhagem, a variável 'saúde' também se mostrou significativa $\left(\mathrm{G}^{2}=18,05\right.$ e sig $\left.=0,00\right)$, assim como a 'cultura' $\left(\mathrm{G}^{2}=11,91\right.$; $\operatorname{sig}=0,03)$, o 'urbanismo' $\left(\mathrm{G}^{2}=9,163\right.$; sig = 0,010) e o 'saneamento' $\left(\mathrm{G}^{2}\right.$ $=27,930$; sig $=0,000$ ), indicando que esses gastos sociais afetam significativamente o crescimento econômico. Em contrapartida, a variável 'educação' não apresentou um efeito significativo $\left(\mathrm{G}^{2}=3,75\right.$; $\left.\operatorname{sig}=0,153\right)$ para o intervalo de confiança considerado.

As estimativas para os parâmetros do modelo estão descritas na tabela 4. Considerando a categoria de referência como a 'baixo' $(P=0)$, as funções logit para as faixas 'médio' e 'alta' são dadas pelas seguintes equações:

$$
\begin{aligned}
& \mathrm{P}(\mathrm{Y}=\text { 'médio' })=-0,748-018 \mathrm{x}_{1}+0,003 \mathrm{x}_{2}-0,004 \mathrm{x}_{3}+0,00 \mathrm{x}_{4}+ \\
& 0,004 \mathrm{x}_{5}+0,007 \mathrm{x}_{6} \\
& \mathrm{P}(\mathrm{Y}=\text { 'alto' })=-1,893-0,004 \mathrm{x}_{1}+0,005 \mathrm{x}_{2}-0,016 \mathrm{x}_{3}-0,002 \mathrm{x}_{4}+ \\
& 0,003 \mathrm{x}_{5}+0,013 \mathrm{x}_{6}
\end{aligned}
$$




\section{Revista de Gestão Pública}

PRÁTICAS E DESAFIOS

ISSN: 2177-1243

TABELA 4 - Estimativas do parâmetro

\begin{tabular}{|c|c|c|c|c|c|c|c|c|c|}
\hline & \multirow{2}{*}{ FAIXA PIBA } & \multirow{2}{*}{ B } & \multirow{2}{*}{$\begin{array}{c}\text { ERRO } \\
\text { PADRÃO }\end{array}$} & \multirow{2}{*}{ WALD } & \multirow{2}{*}{ DF } & \multirow{2}{*}{ SIG. } & \multirow{2}{*}{$\operatorname{EXP}(B)$} & \multicolumn{2}{|c|}{ INTERVALO DE CONFIANÇA $95 \%$ PARA EXP(B) } \\
\hline & & & & & & & & LIMITS INEEPIOP & HIMTE CUPEPIOP \\
\hline \multirow{7}{*}{ MÉDIO } & Interceptação &,- 748 & ,289 & 6,710 & 1 & 010 & & & \\
\hline & Assistência social &,- 018 & ,004 & 23,229 & 1 & ,000 & ,982 & ,975 & ,989 \\
\hline & Saúde & ,003 & ,001 & 8,763 & 1 & ,003 & 1,003 & 1,001 & 1,005 \\
\hline & Cultura &,- 004 & ,003 & 1,406 & 1 & ,236 & ,996 & ,989 & 1,003 \\
\hline & Educação & ,000 & ,001 & ,018 & 1 & ,894 & 1,000 & ,998 & 1,002 \\
\hline & Urbanismo & ,004 & ,001 & 8,814 & 1 & ,003 & 1,004 & 1,001 & 1,007 \\
\hline & Saneamento & ,007 & ,002 & 9,030 & 1 & ,003 & 1,007 & 1,003 & 1,012 \\
\hline \multirow{7}{*}{ ALTO } & Interceptação & $-1,893$ & 347 & 29,763 & 1 & ,000 & & & \\
\hline & Assistência social &,- 004 & ,004 & 1,001 & 1 & 317 & ,996 & ,988 & 1,004 \\
\hline & Saúde & ,005 & ,001 & 16,105 & 1 & ,000 & 1,005 & 1,003 & 1,008 \\
\hline & Cultura &,- 016 & ,005 & 10,493 & 1 & ,001 & ,984 & ,974 & ,994 \\
\hline & Educação &,- 002 & ,001 & 3,019 & 1 & ,082 & ,998 & ,996 & 1,000 \\
\hline & Urbanismo & ,003 & ,002 & 2,341 & 1 & 126 & 1,003 & ,999 & 1,006 \\
\hline & Saneamento & ,013 & ,003 & 24,641 & 1 & ,000 & 1,013 & 1,008 & 1,018 \\
\hline
\end{tabular}

a. A categoria de referência é: baixo.

FONTE: Resultados da pesquisa. 
Os resultados obtidos apontam que os gastos com educação não apresentam efeitos estatisticamente significativo sobre o crescimento econômico dos municípios para o intervalo de confiança considerado $(\operatorname{sig}=0,05)$, ao contrário dos achados de Cruz et al. (2010), que identificaram uma relação positiva da educação com a produtividade da economia. No entanto, vale lembrar que os autores realizaram uma análise temporal dos gastos, o que pode explicar essa significância. Os demais gastos sociais analisados apresentaram efeitos estatisticamente significativos sobre o crescimento do produto.

As despesas com assistência social e cultura impactaram negativamente no crescimento do PIB, indicando que os dispêndios nessas duas áreas contribuem para a diminuição do produto. Isso pode estar relacionado as contribuições de Devarajan et al. (1996) que indicaram que aumentos excessivos nos gastos públicos podem torná-los improdutivos, no entanto, para avaliar isso seria necessário fazer uma análise temporal das despesas. Já as despesas com saúde, urbanismo e saneamento impactaram positivamente no crescimento econômico, $\mathrm{o}$ que vai ao encontro com os achados de Cruz et al. (2010) que também encontraram uma relação positiva entre a saúde e a produtividade da economia. Em contrapartida, os resultados vão de encontro com o estudo de Morais et al. (2012), que encontraram uma relação negativa entre as despesas com saúde e saneamento e o crescimento econômico.

A associação negativa entre algumas despesas e o crescimento econômico dos municípios pode estar relacionada aos resultados encontrados por Pang \& Herrera (2005), que apontaram uma correlação negativa entre a eficiência e o tamanho das despesas. Sendo assim, despesas que estão sendo realizadas de maneira excessiva podem apresentar baixa eficiência e, consequentemente, impactar de maneira negativa na economia. Por outro lado, também há de se considerar que, a tendência de cortes de gastos no setor público pode contribuir para a queda da qualidade dos serviços prestados e assim, um efeito negativo também irá surgir. Nesse sentido, torna-se relevante um estudo mais aprofundado sobre as causas e fontes desses problemas para analisar o porquê desse efeito.

Além disso, Alonso (1999) chama a atenção para o fato do Brasil não apresentar sistemas que permitam avaliar claramente os efeitos gerados pelas ações governamentais, prejudicando a identificação de fontes de desperdícios de recursos bem como onde estes estão melhor alocados. Na mesma linhagem, Cândido Jr. (2001) explica que para avaliar a produtividade dessas despesas é necessário se concentrar nos objetivos principais de cada programa para evitar dispersões e investir naqueles setores que geram maiores externalidade positivas. Dessa forma, é possível afirmar que existem diversos fatores que podem impactar na produtividade dos gastos, sendo que, a má alocação e o desperdício são os principais apontados na literatura.

Identificada a relação entre o PIB e os gastos sociais, buscou-se testar se os diferentes grupos em função do crescimento econômico 
'baixo', 'médio' e 'alto' são iguais em termos de distribuição ou tendência central dos gastos sociais. Para isso, foi empregado o teste de Kruskal-Wallis que visa verificar a hipótese de igualdade em relação à algum parâmetro de localização (PESTANA E GAGEIRO, 2008). Sendo assim, foi definida a seguinte hipótese geral:

$\mathrm{H}_{\mathrm{o}}=$ a distribuição dos gastos sociais é a mesma entre as diferentes faixas do PIB

$$
\mathrm{H}_{1} \text { = contrário. }
$$

Considerando um intervalo de confiança de $5 \%$, os resultados apontaram que as despesas per capita com assistência social $(\operatorname{sig}=0,00)$, saúde $(\operatorname{sig}=0,00)$, cultura $(\operatorname{sig}=0,035)$, urbanismo $(\operatorname{sig}=0,00)$ e saneamento $(0,00)$ permitem rejeitar a hipótese nula e indicam que existem evidências estatísticas de que os três grupos (baixo, médio e alto) apresentam diferentes distribuições em relação a esses gastos. Já o teste para a despesa per capita com educação $(\operatorname{sig}=0,566)$ não permite rejeitar a hipótese nula, o que leva à conclusão de que a distribuição dos gastos com educação per capita é a mesma para todas as faixas de crescimento econômico. Talvez seja um dos motivos pelos quais a educação não se mostrou significativa para explicar o crescimento econômico, uma vez que os municípios estão apresentando a mesma tendência de gastos nesta função.

Ao se analisar a média dos gastos sociais em função das faixas de crescimento econômico, a assistência social per capita apresentou uma média de $\mathrm{R} \$ 49,50$ na faixa 'baixo', $\mathrm{R} \$ 41,38$ na faixa 'médio' e $\mathrm{R}$ \$ 54,47 na faixa 'alto', o que pode ser relacionado com relação negativa entre os dois, uma vez que a faixa 'baixo' apresentou um valor maior que o 'médio', o que pode levar a hipótese dos gastos excessivos se tornarem improdutivos. Já as despesas per capita com saúde apresentaram uma média de $\mathrm{R} \$$ 323,99 para a faixa 'baixo', $\mathrm{R} \$ 354,11$ para o 'médio' e $\mathrm{R} \$$ 391,85 no 'alto', o que justifica a relação positiva entre esses gastos e crescimento econômico, como pode-se visualizar que quanto maior o nível de crescimento maior as despesas com saúde. Quanto à despesa per capita com educação, a média para a faixa de crescimento 'baixo' foi de $\mathrm{R} \$ 356,51$, o 'médio' foi de $\mathrm{R} \$ 371,40$ e o 'alto' 379,01. No entanto, não se pode fazer inferências sobre o seu comportamento em relação ao PIB per capita pelo fato de não ter se mostrado significativa.

Quanto aos gastos com cultura per capita, a média foi de $\mathrm{R} \$ 28,39$, $\mathrm{R} \$ 28,56$ e 24,94 para as faixas 'baixo', 'médio' e 'alto', respectivamente. Aqui também se pôde observar como o nível de maior crescimento do produto apresentou menor valor da despesa, o que justifica sua relação negativa com o crescimento econômico. Ao contrário, a média dos gastos per capita com urbanismo justificam a sua relação positiva com o crescimento econômico, uma vez que as faixas apresentaram valores crescentes para estas despesas, de $\mathrm{R} \$ 104,93, \mathrm{R} \$ 123,63$ e $\mathrm{R} \$ 131,34$, respectivamente. $\mathrm{O}$ mesmo pode ser observado sobre os gastos per capita com 
saneamento, que apresentaram média para os níveis 'baixo', 'médio' e 'alto' de $\mathrm{R} \$ 24,15, \mathrm{R} \$ 34,88$ e $\mathrm{R} \$ 51,24$, respectivamente, justificando sua relação positiva com o produto. Os achados podem ser associados a "Lei dos Dispêndios Públicos Crescentes" proposta por Wagner (1980) apud Cândido Jr. (2001), que aponta que um dos fatores que contribuem para o crescimento da economia seria uma maior participação do governo na provisão de bens públicos, o que pode ser visualizados nesses gastos sociais crescentes.

\section{CONCLUSÕES}

Com bases nos resultados apresentados, conclui-se que investimentos nas áreas de saúde, urbanismo e saneamento contribuem para o crescimento econômico, corroborando com o trabalho de Cruz et al. (2010), ao contrário de Morais et al. (2012), que identificaram uma associação negativa. Ademais, pôde-se observar que investimentos em assistência social e cultura ocasionam uma diminuição do PIB. A análise dos grupos de municípios por faixa de crescimento econômico permitiu confirmar tais proposições, uma vez que o primeiro conjunto de despesas apresentaram uma tendência crescente em função do nível de crescimento econômico, enquanto que o segundo conjunto apresentou uma tendência decrescente, o que permite concluir que quanto maior a carência assistencial da população menor a sua disponibilidade financeira para adquirir bens e serviços.

O trabalho buscou contribuir para as discussões em torno dos efeitos dos gastos públicos sobre o crescimento da economia. Sendo assim, os achados podem orientar decisões políticas acerca da alocação de recursos públicos em áreas que apresentam relação com o crescimento ou decrescimento do PIB. Como as despesas com saúde, urbanismo e saneamento foram as que apresentaram efeitos positivos sobre o produto, isto pode indicar uma possível necessidade de priorizar esses gastos por parte do governo. Além disso, a pesquisa também desperta o interesse para se investigar a qualidade desses gastos por parte dos gestores públicos, uma vez que, assim, é possível fazer melhores inferências sobre seus impactos. E por fim, os resultados também reforçam as proposições da base teórica utilizada no artigo, principalmente, no que tange a importância da boa gestão associada a produtividade dos gastos sociais para que eles possam ser considerados produtivos.

Como limitações da pesquisa, buscou-se identificar a relação existente entre os gastos sociais e o crescimento econômico e não a qualidade destes gastos, bem como a análise de sua eficiência. Além disso, o estudo foi realizado por meio de um cross-section para apenas um ano das despesas em relação ao PIB, o que não permite analisar a sua tendência de crescimento para confirmar algumas proposições teóricas apontadas sobre tamanho e possíveis excessos de gastos. Sendo assim, sugere-se para novos estudos, realizar uma análise temporal das despesas sociais para analisar o seu comportamento no decorrer dos anos. Além disso, a pesquisa pode ser expandida para outras unidades da 
federação, bem como novas variáveis podem ser incorporadas para melhorar a qualidade de ajuste do modelo. Mesmo assim, vale lembrar que o artigo cumpre o seu objetivo de identificar a existência de relação entre os dois fenômenos analisados, não apresentando pretensões explicativas das relações de causa e efeito.

\section{REFERÊNCIAS BIBLIOGRÁFICAS}

ALONSO, M. Custos no serviço público. Revista do Serviço Público, v. 50, n. 1, p. 37-63, 1999.

AFONSO, J. R. R.; ARAÚJO, E. A. A capacidade de gasto dos municípios brasileiros: arrecadação própria e receita disponível. BNDES, Rio de Janeiro, 2001. .

BITTENCOURT, H. R. Regressão Logística Politômica: Revisão Teórica e Aplicações. Acta Scientia, Canoas, v. 5, n. 1, p. 77-86, 2003.

CÂNDIDO JR., J. O. Os gastos públicos no Brasil são produtivos? Pesquisa e Planejamento Econômico, Ipea, n. 23, jun. 2001.

COX, D. R.; SNELL, E. J. The Analysis of Binary

Data. London: Chapman and Hall, 1989.

CRUZ, A. C.; TEIXEIRA, E. C.; BRAGA, J. M. Os efeitos dos gastos públicos em infraestrutura e em capital humano no crescimento econômico e na redução da pobreza no Brasil. Economia, Selecta, v. 11, n. 4, p. 163-185, 2010.

DEVARAJAN, S.; SWARROP, V.; ZOU, H. The composition of public expenditure and economic growth. Journal of Monetary Economics, v. 37, p. 313-344, 1996.

FELDSTEIN, M. Government deficits and aggregate demand. Journal of Monetary Economics, v. 9, n. 1, p. 1-20, 1982.

FINANÇAS DO BRASIL. Dados contabéis dos municípios. Disponível em: < http://www.tesouro.fazenda. gov.br/contas-anuais> Acesso em: 8 dez. 2016.

FUNDAÇÃO JOÃO PINHEIRO. Perfil de Minas Gerais, $15^{\underline{a}}$ edição, 2012. Disponível em: <http://www.fjp.mg.gov.br/index. php/docman/cei/perfil-de-minas-gerais/128-perfil-de-minasgerais-2012-portugues-ingles/file> Acesso em: 8 dez. 2016.

HERRERA, S.; BLANCO, F. The quality of fiscal adjustment and the long run growth impact of fiscal policy in Brazil. Mimeo, 2004.

HOSMER, D.; LEMESHOW, S. Applied Logistic

Regression. New York: John Wiley \& Sons, 1989.

INSTITUTO BRASILEIRO DE GEOGRAFIA E ESTATÍSTICA. Produto Interno Bruto dos municípios. IBGE, Rio de Janeiro, 2010. Disponível em: < http://www.fjp.mg.gov.br/index.php/ docman/cei/pib/pib-municipais> Acesso em: 8 dez. 2016. 
MAROCO, J. Análise estatística: com utilização do SPSP. $5^{\underline{a}}$ edição, Pero Pinheiro: Report Number, 2011.

MORAIS, G. S; ARAÚJO, J. A.; MONTEIRO, V. B. Gastos Públicos e Crescimento Econômico: evidências da economia cearense. Encontro do Instituto de Pesquisa e Estratégia Econômica do Ceará, IPECE, 2012.

MOTTA, R. S.; MOREIRA, A. Eficiência na gestão municipal no Brasil. Texto para discussão do Ipea, n. 1301, 2007.

PANG, G.; HERRERA, S. Efficiency of public spending in developing countries: an efficiency frontier approach. World Bank Policy Research Working Paper, n. 3645, 2005.

PESTANA, M. H.; GAGEIRO, J. N. Análise de dados para ciências sociais: a complementaridade do SPSS, 5ae edição, Lisboa: Sílabo, 694p, 2008.

REZENDE, F. C. Descentralização, gastos públicos e preferências alocativas dos governos locais no Brasil (1980-1994). Dados, v. 40, n. 3, p. 441-464, 1997.

RIBEIRO, M. B. Desempenho e eficiência do gasto público: uma análise comparativa entre o Brasil e um conjunto de países da América Latina. Rio de Janeiro: Ipea, 2008.

ROCHA, F.; GIUBERT, A. C. Composição do gasto público e crescimento econômico: uma avaliação macroeconômica da qualidade dos gastos dos estados brasileiros.

Economia Aplicada, v. 11, n. 4, p. 463-485, 2007.

TRUETT, J.; CORNFIELD, J.; KANNEL, W. A multivariate analysis of the risk of coronary heart disease in Framinghan. Journal of Chronic Diseases. v. 20, p. 511-524, 1967.

WAGNER, A. Finanzwissenschaft. Leipzig, 1890. 